\title{
Resumen guía de práctica clínica para la valoración urológica pretrasplante renal
}

\section{Resumed Clinical Practice Guidelines, Urological Evaluation for Kidney Transplant}

Diego Riatiga Ibáñez, MD, MSc 1,2,3,4 Jorge Cubillos, MD5,6,7,8

Verónica Tovar Roa, MD, MSc 9,10,11,12,13,14 William Ricardo Fajardo Cediel, MD 15,16,17,18,19

Herney Andrés García Perdomo, MD, PhD 20,21,22,23,24

${ }^{1}$ Médico y Cirujano, Especialista en Urología, Profesor y Investigador,

Fundación Universitaria de Ciencias de la Salud, Colombia

2 Master Universitario en Nefrología Diálisis y Trasplante Renal,

Universidad San Jorge Zaragoza, España

${ }^{3}$ Coordinador de línea de investigación de Urologia Clínicoquirúrgica, Grupo de investigación NEFROUROLOGIA DIÁLISIS Y

TRASPLANTE (Colciencias B), Bogotá, Colombia

${ }^{4}$ Jefe del Servicio de Urología Hospital Infantil Universitario de San José, Colombia; Sección de Trasplante Renal de la Sociedad Colombiana de Urología, Bogotá, Colombia

${ }^{5}$ Médico General y Urólogo, Universidad Estatal de Vinnitsa, Ucrania

${ }^{6}$ Cirujano Trasplante Renal, Hospital 12 de Octubre de Madrid,

España

${ }^{7}$ Especialista en Gerencia de Servicios de Salud, Universidad Cooperativa de Colombia

${ }^{8}$ Director, Grupo de Investigación, Fundación Surcolombiana de Transplantes, (TTS y ESOT), Bogotá, Colombia

${ }^{9}$ Médico Universidad Autónoma de Bucaramanga

${ }^{10}$ Especialista en Urología, Pontificia Universidad Javeriana

11 Máster Universitario de investigación clínica en Donación y Trasplante de Órganos, Tejidos y Células

12 Universidad de Barcelona, España

13 Docente de Universidad Autónoma de Bucaramanga; Uróloga y Cirujana de Trasplante Renal

\footnotetext{
${ }^{14}$ FOSCAL - Centro Urológico, Bucaramanga, Colombia

15 Médico Cirujano, Universidad Nacional de Colombia

16 Especialista en Urología, Universidad El Bosque

${ }^{17}$ Entrenamiento en Trasplante Renal (Renal, Páncreas e Hígado), Bogotá, Colombia

${ }^{18}$ Cirujano de Trasplante Renal; Unidad de Trasplantes, Fundación Clínica Shaio, Bogotá, Colombia

${ }^{19}$ Entrenamiento en Trasplante Renal, Fairview Hospital, University of Minnesota, Minneapolis, USA

20 Médico y Cirujano, Especialista en Urología, Profesor Asociado e Investigador, Universidad del Valle, Chile

${ }^{21}$ Magíster en Epidemiología Clínica Universidad de la Frontera, Chile

22 Doctor en Educación Universidad Autónoma de Durango, Mexico

${ }^{23}$ Doctor en Medicina Clínica y Salud Pública Universidad de Granada, España

${ }^{24}$ Director, Grupo de Investigación UROGIV y Grupo Asociado Cochrane - Universidad del Valle, Valle del Cauca, Colombia
}

Address for correspondence Diego Riatiga Ibáñez, MD, Fundación Universitaria de Ciencias de la Salud, Colombia

(e-mail: dr.riatiga@gmail.com; guiaseinvestigacion@scu.org.co).

Urol Colomb 2018;27:3-7.
Resumen
Palabras-Clave
- guía
- valoración
- pretrasplante renal

En Colombia actualmente, se ha visto un aumento significativo en el número de pacientes con enfermedad renal crónica terminal (ERC-T) llegando a 23.914 en 2014 según las estadísticas del instituto nacional de salud, lo que supone un aumento en el número de trasplantes, aumentando de un 12,5\% en 2008 al 18\% en 2014 del total de pacientes con ERC-T. Teniendo en cuenta que el trasplante renal se ha consolidado como la mejor opción terapéutica en esos pacientes, es de suma importancia unificar criterios y conceptos basados en la mejor evidencia científica disponible para reducir complicaciones y efectos nocivos derivados de esta terapia, razón por la cual se ha creado esta guía.
DOI https://doi.org/ $10.1055 / \mathrm{s}-0038-1636526$ ISSN $0120-789 X$ eISSN 2027-0119.
Copyright ( 2018, Sociedad Colombiana License terms de Urología. Publicado por Thieme Revinter Publicações Ltda., Rio de Janeiro, Brazil. Todos los derechos reservados. 


\begin{abstract}
Keywords

- guideline

- validation

- kidney transplant

In Colombia, exist a significant increase in the number of patients with terminal chronic kidney disease (CKD), up to 23,914 patients in 2014 according to statics of the national health institute. This means an increase in the number of transplants, from $12.5 \%$ in 2008 to $18 \%$ in 2014 from the overall patients with terminal CKD. Considering that kidney transplant is the best option to those patients, it became of extreme importance to unify criteria and concepts based in the best available evidence to reduce complications and negative effects from the therapy. For these reasons we have created this guideline.
\end{abstract}

\section{Introducción}

En Colombia actualmente se ha visto un aumento significativo en el número de pacientes con enfermedad renal crónica terminal (ERC-T) llegando a 23.914 en 2014 según las estadísticas del instituto nacional de salud, lo que supone un aumento en el número de trasplantes, aumentando de un $12,5 \%$ en 2008 al $18 \%$ en 2014 del total de pacientes con ERC-T. Teniendo en cuenta que el trasplante renal se ha consolidado como la mejor opción terapéutica en esos pacientes, es de suma importancia unificar criterios y conceptos basados en la mejor evidencia científica disponible para reducir complicaciones y efectos nocivos derivados de esta terapia, razón por la cual se ha creado esta guía.

\section{Objetivos}

\section{General}

Establecer pautas unificadas para la evaluación urológica pretrasplante.

\section{Objetivos Específicos}

- Especificar las principales recomendaciones para la evaluación urológica en pacientes con intención de donación viva.

- Estandarizar pautas para la preservación del injerto renal durante el proceso de trasplante

- Establecer recomendaciones claras para la evaluación pretrasplante de los pacientes receptores de trasplante renal.

\section{Metodología}

La presente guía es una adaptación y actualización de la guía de la Asociación Europea de Urología (EAU) de trasplante renal. Está actualizada hasta febrero de 2016 en las siguientes bases de datos: CENTRAL, DARE, EMBASE, HTA Y MEDLINE.

\section{Registro de conflicto de interés de los miembros del grupo}

Todos los miembros del grupo, así como las personas que han participado tanto en la colaboración experta como en la revisión externa, declaran que no hay conflictos de interés. Además no ha habido financiación externa para el desarrollo del presente documento.

\section{Aplicabilidad}

La población a la que va dirigida la presente guía es cualquier persona que vaya a ser sometida a un trasplante renal y por tanto requiera una valoración clínica previo a su procedimiento, así como a los potenciales donantes vivos, como también a todo el personal medico que forma parte de este proceso.

\section{Cada cuánto tiempo se hará la actualización}

De acuerdo con la política de la Sociedad Colombiana de Urología se hará la revisión cada 2 años y la actualización a cada 5 años.

\section{El donante vivo}

En la actualidad, el $20 \%$ de todos los trasplantes renales en EUROTRANSPLANT y el $40 \%$ en EE.UU. se realizan con donantes vivos $(14,16)$ (grado de comprobación científica: 2a) (-Tablas 1 y 2).

Tabla 1 Ventajas de la donación en vida

\begin{tabular}{|l|}
\hline $\begin{array}{l}\text { - Mejores resultados (a corto y largo plazo) en } \\
\text { comparación con los injertos de donantes fallecidos }\end{array}$ \\
\hline - Función precoz sistemática y tratamiento más fácil \\
\hline $\begin{array}{l}\text { - Disminución de un tiempo largo de espera para el } \\
\text { trasplante }\end{array}$ \\
\hline - Regímenes inmunosupresores menos agresivos \\
\hline - Ganancia emocional para el donante \\
\hline - Aumento mundial de la tasa de trasplantes renales \\
\hline
\end{tabular}

Tabla 2 Criterios de exclusión de donantes vivos

\begin{tabular}{|l|}
\hline Contraindicaciones absolutas \\
\hline - Edad $<18$ años \\
\hline - Hipertensión arterial no controlada \\
\hline - Diabetes mellitus \\
\hline - Proteinuria $(>300 \mathrm{mg} / 24 \mathrm{~h})$ \\
\hline - FG anormal para la edad \\
\hline - Hematuria \\
\hline - Riesgo alto de tromboembolia \\
\hline
\end{tabular}


Tabla 2 (Continued)

\begin{tabular}{|l|}
\hline Contraindicaciones absolutas \\
\hline $\begin{array}{l}\text { - Enfermedad importante (enfermedad pulmonar crónica, } \\
\text { tumor maligno reciente, cardiopatía) }\end{array}$ \\
\hline - Infección por el VIH \\
\hline Contraindicaciones relativas \\
\hline $\begin{array}{l}\text { - Infección activa crónica (por ejemplo, tuberculosis, } \\
\text { hepatitis B/C o parásitos) }\end{array}$ \\
\hline - Obesidad \\
\hline - Trastornos psiquiátricos \\
\hline - Antecedente de enfermedad litiásica (se recomienda \\
individualizar a cada paciente)
\end{tabular}

Abreviaciones: FG, filtración glomerular; VIH, virus de la inmunodeficiencia humana.

\section{Elección del riñón}

Siempre debe dejarse el 'mejor' riñón al donante.

\begin{tabular}{|l|l|}
\hline Recomendaciones & GR \\
\hline $\begin{array}{l}\text { El uso de donantes vivos se ha asociado a tasas } \\
\text { de éxito más altas que las observadas con la } \\
\text { donación de donantes cadavéricos. La } \\
\text { donación en vida permite que algunos } \\
\text { pacientes eviten tiempos de espera } \\
\text { prolongados e incluso la diálisis. }\end{array}$ & \\
\hline $\begin{array}{l}\text { En todos los casos es obligatoria } \\
\text { una evaluación independiente } \\
\text { de la función renal del donante }\end{array}$ & \\
por un nefrólogo o un equipo & \\
especializado. & B \\
\hline $\begin{array}{l}\text { Se recomienda obtener una evaluación } \\
\text { psiquiátrica o médica independiente de la } \\
\text { motivación del donante, su forma física y su } \\
\text { capacidad para comprender los riesgos de la } \\
\text { intervención. }\end{array}$ & \\
\hline $\begin{array}{l}\text { Es responsabilidad del cirujano urólogo } \\
\text { garantizar que el donante sea adecuado } \\
\text { desde el punto de vista médico para } \\
\text { el procedimiento, que el órgano donado } \\
\text { se encuentra sano y que la expectativa de } \\
\text { éxito en el receptor es razonable. }\end{array}$ & \\
\hline $\begin{array}{l}\text { Al donante siempre se le debe dejar el ‘mejor’ } \\
\text { riñón. La extirpación renal mediante un } \\
\text { abordaje transperitoneal entraña un } \\
\text { mayor número de complicaciones } \\
\text { esplénicas e intestinales que otras opciones } \\
\text { quirúrgicas. }\end{array}$ & \\
\hline $\begin{array}{l}\text { La nefrectomía abierta del donante } \\
\text { ha de realizarse mediante un } \\
\text { abordaje extraperitoneal a través } \\
\text { de una incisión subcostal o lumbotomía dorsal. }\end{array}$ & \\
\hline $\begin{array}{l}\text { La nefrectomía laparoscópica del donante } \\
\text { trans o retroperitoneal) sólo debe ser } \\
\text { realizada por personas con formación en el } \\
\text { procedimiento. }\end{array}$ & \\
\hline $\begin{array}{l}\text { La nefrectomía laparoscópica con asistencia } \\
\text { manual del donante reduce al mínimo el tiempo } \\
\text { de isquemia caliente en comparación con las } \\
\text { otras intervenciones laparoscópicas clásicas. }\end{array}$ & \\
\hline
\end{tabular}

\section{Conservación del órgano}

\begin{tabular}{|l|l|}
\hline Recomendaciones & GR \\
\hline $\begin{array}{l}\text { Las soluciones UW (Universidad de Wisconsin) } \\
\text { y HTK (histidina-triptófano-alfacetoglutara- } \\
\text { to) son soluciones de conservación de } \\
\text { referencia y tienen la misma eficacia en } \\
\text { donantes multiorgánicos o de un solo riñón. }\end{array}$ & \\
\hline La solución Celsior parece igual de eficaz. & B \\
\hline $\begin{array}{l}\text { En todo trasplante renal hay que mantener } \\
\text { unos tiempos de isquemia fría y caliente lo } \\
\text { más breves posibles. }\end{array}$ & A \\
\hline
\end{tabular}

Abreviaciones: GR, grado de recomendación.

\section{Receptor Renal}

\begin{tabular}{|l|l|}
\hline Recomendación & GR \\
\hline Una evaluación preoperatoria completa de & B \\
todos los candidatos a trasplante es & \\
obligatoria para mejorar la supervivencia del & \\
órgano y el paciente en el período & \\
postrasplante. Dicha evaluación ha de & \\
repetirse con regularidad. & \\
\hline
\end{tabular}

Abreviaciones: GR, grado de recomendación.

\section{Tratamiento antes del trasplante}

\begin{tabular}{|l|l|}
\hline Recomendaciones & GR \\
\hline $\begin{array}{l}\text { En caso de un aparato genitourinario anormal, } \\
\text { se requiere una evaluación meticulosa antes } \\
\text { del trasplante, siendo las pruebas } \\
\text { urodinámicas la investigación clave. }\end{array}$ & \\
\hline $\begin{array}{l}\text { Cuando el tratamiento farmacológico o el } \\
\text { cateterismo intermitente fracasa o no es } \\
\text { posible, resulta necesaria una derivación } \\
\text { urinaria mediante reservorios cateterizables, } \\
\text { conductos o cistoplastias. }\end{array}$ & \\
\hline $\begin{array}{l}\text { La PRAD con espacio insuficiente o } \\
\text { complicaciones, infecciones crónicas o } \\
\text { riñones con sospecha de crecimiento tumoral, } \\
\text { deben extirparse de forma preoperatoria o } \\
\text { concomitante con el trasplante. }\end{array}$ & \\
\hline $\begin{array}{l}\text { Una infección activa, que puede agravarse } \\
\text { después del trasplante y causar una infección } \\
\text { potencialmente mortal, es una } \\
\text { contraindicación del trasplante. }\end{array}$ & B \\
\hline $\begin{array}{l}\text { Cribado de enfermedades víricas y bacterianas } \\
\text { en todos los candidatos a trasplante Cribado } \\
\text { de todos los pacientes de IVU previas, VHB, } \\
\text { VHC, VIH, CMV y TB (anamnesis y radiografía } \\
\text { de tórax). }\end{array}$ & \\
\hline $\begin{array}{l}\text { El cribado sistemático de todos los pacientes } \\
\text { en todas las subespecialidades no resulta } \\
\text { necesario. }\end{array}$ & B \\
\hline $\begin{array}{l}\text { En caso de comorbilidad grave o } \\
\text { incumplimiento terapéutico, debe realizarse } \\
\text { una evaluación minuciosa e individualizada. }\end{array}$ & \\
\hline & C \\
& \\
\hline
\end{tabular}

(Continued) 
(Continued)

\begin{tabular}{|c|c|}
\hline Recomendaciones & GR \\
\hline $\begin{array}{l}\text { La evaluación previa al trasplante debe } \\
\text { centrarse en la presencia de cardiopatías. }\end{array}$ & $B$ \\
\hline $\begin{array}{l}\text { En los pacientes con un riesgo elevado de } \\
\text { cardiopatía, se recomienda encarecidamente } \\
\text { una evaluación exhaustiva para descartar } \\
\text { enfermedad coronaria. }\end{array}$ & B \\
\hline $\begin{array}{l}\text { La revascularización ha de practicarse antes } \\
\text { del trasplante. }\end{array}$ & B \\
\hline $\begin{array}{l}\text { Durante la evaluación previa al trasplante, hay } \\
\text { que prestar especial atención a la enfermedad } \\
\text { ilíaca, periférica y cerebrovascular. Se } \\
\text { recomienda tomar las medidas diagnósticas y } \\
\text { terapéuticas pertinentes. }\end{array}$ & $\mathrm{C}$ \\
\hline $\begin{array}{l}\text { Los pacientes con diabetes mellitus deben } \\
\text { someterse a un trasplante. Requieren una } \\
\text { evaluación exhaustiva antes del trasplante. }\end{array}$ & B \\
\hline $\begin{array}{l}\text { La obesidad no es una contraindicación del } \\
\text { trasplante. Sin embargo, se recomienda una } \\
\text { evaluación exhaustiva previa al trasplante y un } \\
\text { intento de reducir peso. }\end{array}$ & $\mathrm{C}$ \\
\hline $\begin{array}{l}\text { Se recomienda una evaluación cuidadosa de } \\
\text { las coagulopatías en los pacientes de riesgo } \\
\text { con el fin de prevenir episodios trombóticos } \\
\text { precoces después del trasplante. }\end{array}$ & C \\
\hline $\begin{array}{l}\text { Las enfermedades que podrían } \\
\text { influir en la evolución posterior al trasplante } \\
\text { deben identificarse } \\
\text { durante la evaluación previa y, si es posible, } \\
\text { tratarse antes del } \\
\text { trasplante. }\end{array}$ & C \\
\hline $\begin{array}{l}\text { Aunque la edad no es una contraindicación del } \\
\text { trasplante, se necesita una evaluación } \\
\text { minuciosa antes del mismo. Debe realizarse } \\
\text { una evaluación cuidadosa de riesgos y } \\
\text { beneficios y asesorar al paciente sobre los } \\
\text { mayores riesgos relacionados con la edad. }\end{array}$ & B \\
\hline $\begin{array}{l}\text { La recidiva de la enfermedad original es } \\
\text { habitual, pero la pérdida del injerto por } \\
\text { recidiva es infrecuente. }\end{array}$ & $C$ \\
\hline $\begin{array}{l}\text { Tan sólo algunas enfermedades raras con una } \\
\text { tasa elevada de recidivas que conllevan a una } \\
\text { pérdida precoz del injerto, son una } \\
\text { contraindicación del trasplante renal. }\end{array}$ & C \\
\hline $\begin{array}{l}\text { Los pacientes con riesgo de enfermedades } \\
\text { recurrentes han de recibir asesoramiento } \\
\text { antes del trasplante, especialmente antes de } \\
\text { un trasplante renal de donante vivo } \\
\text { emparentado. }\end{array}$ & $\mathrm{C}$ \\
\hline $\begin{array}{l}\text { La evaluación previa al trasplante en los } \\
\text { pacientes con retrasplante o trasplante } \\
\text { extrarenal previo, debe centrarse en el riesgo } \\
\text { inmunológico, incluido un análisis detallado } \\
\text { para determinar la presencia de anticuerpos } \\
\text { anti-HLA. }\end{array}$ & C \\
\hline
\end{tabular}

\section{Resumen de la evaluación urológica del receptor pretrasplante.}

\section{Anamensis}

- Causa de la ERC-T

- Evaluación de patrón miccional y hábitos miccionales previos

- IVU previas o IVU recurrente

- Episodios previos de hematuria

- Comorbilidades de riesgo urológico (Diabetes mellitus, enfermedades neurológicas o degenerativas, uretritis, cirugías previas etc.)

\section{Examen físico}

- Se recomienda realizar un examen físico completo

- ERD en pacientes mayores de 50 años o con riesgo mayor de cáncer de próstata

- Evaluación genital completa

\begin{tabular}{|l|l|}
\hline Obligatorios & Opcionales \\
\hline Urocultivo / parcial de orina* & Cistoscopia \\
\hline $\begin{array}{l}\text { Ultrasonografía renal y de } \\
\text { vías urinarias }\end{array}$ & Urodinamia estandar \\
\hline $\begin{array}{l}\text { Ecografía dúplex de vasos } \\
\text { iliacos }\end{array}$ & Cistografía miccional \\
\hline PSA ${ }^{* *}$ & $\begin{array}{l}\text { Angio TAC aortoiliaco } \\
\text { (ptes }>40 \text { años) }\end{array}$ \\
\hline
\end{tabular}

\section{Bibliografia}

1 Oosterlee A, Rahmel A, van Zwet W (eds). Annual report 2005. Leiden: Eurotransplant International Foundation; 2005

2 Malaise J, Van Deynse D, Dumont V, et al. Non-heart-beating donor, 10-year experience in a Belgian transplant center. Transplant Proc 2007;39(08):2578-2579

3 Abouna GM. Ethical issues in organ and tissue transplantation. Exp Clin Transplant 2003;1(02):125-138

4 Moritsugu KP. Organ donation: the gift of life. J Am Diet Assoc 2007;107(01):15

5 Sanni AO, Wilson $\mathrm{CH}$, Wyrley-Birch $\mathrm{H}$, et al. Non-heart-beating kidney transplantation: 6-year outcomes. Transplant Proc 2006; 38(10):3396-3397

6 Gok MA, Bhatti AA, Asher J, et al. The effect of inadequate in situ perfusion in the non heart-beating donor. Transpl Int 2005;18 (10):1142-1146

7 Signori S, Boggi U, Vistoli F, et al. Regional procurement team for abdominal organs. Transplant Proc 2004;36(03):435-436

8 Simforoosh N, Bassiri A, Ziaee SA, et al. Laparoscopic versus open live donor nephrectomy: the first randomized clinical trial. Transplant Proc 2003;35(07):2553-2554

9 Dalla Valle R, Mazzoni MP, Capocasale E, et al. Laparoscopic donor nephrectomy: short learning curve. Transplant Proc 2006;38(04): 1001-1002

10 Brown SL, Biehl TR, Rawlins MC, Hefty TR. Laparoscopic live donor nephrectomy: a comparison of the conven- tional open approach. J Urol 2001;165(03):766-769

11 Berardinelli L. Technical problems in living donor transplantation. Transplant Proc 2005;37(06):2449-2450 
12 Ruszat R, Wyler SF, Wolff T, et al. Reluctance over right-sided retroperitoneoscopic living donor nephrectomy: justified or not? Transplant Proc 2007;39(05):1381-1385

13 Sasaki TM, Finelli F, Bugarin E, et al. Is laparoscopic donor nephrectomy the new criterion standard? Arch Surg 2000;135 (08):943-947

14 Dittrich S, Groneberg DA, von Loeper J, et al. Influence of cold storage on renal ischemia reperfusion injury after non-heartbeating donor explantation. Nephron Exp Nephrol 2004;96(03): e97-e102

15 Kootstra G, Daemen JH, Oomen AP. Categories of non-heart beating donors. Transplant Proc 1995;27(05):2893-2894

16 Azar SA, Nakhjavani MR, Tarzamni MK, Faragi A, Bahloli A, Badroghli N. Is living kidney donation really safe? Transplant Proc 2007;39(04):822-823

17 Hazebroek EJ, Gommers D, Schreve MA, et al. Impact of intraoperative donor management on short-term renal function after laparoscopic donor nephrectomy. Ann Surg 2002;236(01):127-132

18 Lucan M (ed). [Textbook of surgical urologic techniques.] Bucharest: Infomedica; 2001:528-536

19 Signori S, Boggi U, Vistoli F, et al. Regional procurement team for abdominal organs. Transplant Proc 2004;36(03):435-436

20 Gres P, Avances C, Iborra F, Mourad G, Guiter J. Long-term morbidity of living donor kidney harvesting. Prog Urol 2007;17 (02):194-198
21 Gürke L, Steiger J, Gasser TC, Sulser T, Bachmann A. Reluctance over right-sided retroperitoneoscopic living donor nephrectomy: justified or not? Transplant Proc 2007;39(05):1381-1385

22 Sasaki TM, Finelli F, Bugarin E, et al. Is laparoscopic donor nephrectomy the new criterion standard? Arch Surg 2000;135 (08):943-947

23 Dittrich S, Groneberg DA, von Loeper J, et al. Influence of cold storage on renal ischemia reperfusion injury after non-heartbeating donor explantation. Nephron Exp Nephrol 2004;96(03): e97-e102

24 Kootstra G, Daemen JH, Oomen AP. Categories of non-heart beating donors. Transplant Proc 1995;27(05):2893-2894

25 Azar SA, Nakhjavani MR, Tarzamni MK, Faragi A, Bahloli A, Badroghli N. Is living kidney donation really safe? Transplant Proc 2007;39(04):822-823

26 Hazebroek EJ, Gommers D, Schreve MA, et al. Impact of intraoperative donor management on short-term renal function after laparoscopic donor nephrectomy. Ann Surg 2002;236(01):127-132

27 Lucan M (ed). [Textbook of surgical urologic techniques.] Bucharest: Infomedica; 2001:528-536

28 Signori S, Boggi U, Vistoli F, et al. Regional procurement team for abdominal organs. Transplant Proc 2004;36(03):435-436

29 Gres P, Avances C, Iborra F, Mourad G, Guiter J. Long-term morbidity of living donor kidney harvesting. Prog Urol 2007;17 (02):194-198 